\title{
Plano de amostragem para Dichopelmus notus (Eriophyidae) na cultura da erva-mate
}

\author{
Sampling plan for Dichopelmus notus (Eriophyidae) on mate-tea orchard
}

\author{
Guilherme Bertoldo' Alfredo de GouveaII Luis Francisco Angeli Alves ${ }^{\text {II }}$
}

RESUMO

O objetivo deste trabalho foi avaliar a amostragem presença-ausência para Dichopelmus notus Keifer (Eriophyidae) na cultura da erva-mate. O trabalho foi realizado em plantação comercial, em Cascavel, PR, entre abril de 1999 e julho de 2000. Procederam-se a coletas quinzenais de 240 folhas em diferentes partes de 10 plantas. A contagem dos ácaros nas folhas foi realizada, em laboratório, com auxílio de microscópio estereoscópico. Foi determinado o padrão de agregação com o modelo da potência de Taylor, e também a proporção de folhas infestadas e o número de amostras requeridas foram estimados por análise de regressão. $\boldsymbol{D}$. notus apresentou distribuição agregada. A proporção de folhas infestadas estimadas pelo modelo matemático mostrou ser um parâmetro confiável para se estimar a densidade populacional do ácaro, sendo que o número de amostras requeridas foi pequeno, o que viabiliza a aplicação prática do método.

Palavras-chave: amostragem binomial, ácaro, Ilex paraguariensis, modelo de Wilson \& Room (1983).

\section{ABSTRACT}

The objective of this research was to evaluate presence-absence sampling plan for Dichopelmus notus Keifer (Eriophyidae) on mate-tea orchard. This study was conducted in Cascavel, State of Paraná, Brazil, from April 1999 to July 2000 , in a mate-tea tree commercial plantation. Biweekly sampling of 240 leaves in different parts of 10 plants was conducted, and the number of mites was registered. The aggregation pattern was determined using the Taylor's power law model. The proportion of the infested leaves and the number of required samples were estimated by regression analysis. $\boldsymbol{D}$. notus presented aggregated distribution. The estimated proportion of infested leaves showed to be reliable in order to estimate the population density of mites. The number of sample unities required to estimate infestation density was low, which makes practical application of this sampling method to be feasible.

Key words: binomial sampling, mite, Ilex paraguariensis, Wilson \& Room model (1983).

\section{INTRODUÇÃO}

A primeira referência sobre ácaros na cultura da erva-mate (Ilex paraguariensis St. Hil.) no Brasil deve-se a PARSEVAL (1939). O autor constatou que plantas de erva-mate apresentavam manchas de coloração arroxeada nas folhas, principalmente em brotações novas, estando associada a estas manchas a presença de ácaros da família Eriophyidae. Trata-se do ácaro Dichopelmus notus Keifer, conhecido popularmente como microácaro ou ácaro-dobronzeamento da erva-mate, cujos adultos possuem dois pares de pernas, corpo em forma de vírgula, com coloração normalmente marrom, com variações dependendo da maturidade das folhas que lhes servem como alimento. Muito ágeis, podem ser encontrados provocando danos durante o ano todo na cultura. Sua ação provoca o arroxeamento das folhas novas das brotações, retardando seu crescimento e provocando sua queda, afetando diretamente a produção. Essa espécie de ácaro tem sido responsável por danos consideráveis, ocasionados por ataques explosivos e rápidos (COLL \& CACERES, 1995; SANTANA et al., 1997; MENEGUZZI et al., 2002; GOUVEA et al., 2006).

\footnotetext{
IDepartamento de Física, Universidade Federal do Paraná (UFPR), Curitiba, PR, Brasil.

IUniversidade Tecnológica Federal do Paraná (UTFPR), Dois Vizinhos, PR, Brasil.

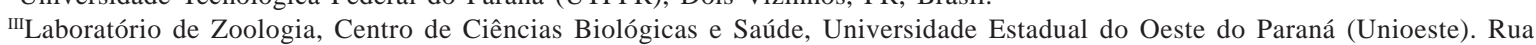
Universitária, 2069, 85819-110, Cascavel, PR, Brasil. Autor para correspondência.
} 
A condução de ervais a pleno sol, na forma de monocultivo, com características distintas das do seu ambiente natural, leva à formação de um agroecossistema de equilíbrio frágil, tornando-se necessário o monitoramento constante dos artrópodes neles presentes, visando à obtenção de informações sobre seus comportamentos, tendo-se em vista seu manejo. A avaliação populacional de $\boldsymbol{D}$. notus pelo método baseado na contagem de indivíduos, comumente empregado na avaliação dos demais artrópodes, apresenta limitações no monitoramento desse eriofiídeo, devido a seu reduzido porte e sua ocorrência em densidades populacionais altas, o que demanda um gasto elevado de tempo e dificulta sua aplicação prática. Adicionalmente, de acordo com RIBEIRO et al. (1990) e PERRUSO \& CASSINO (1997), a contagem pode resultar numa estimativa imprecisa da população, sendo de difícil aplicação prática. A metodologia presença-ausência (binomial), na qual se considera o número de amostras com a presença ou não do ácaro e não o número de indivíduos por amostra, tem sido apresentada como uma alternativa para o monitoramento de organismos semelhantes (WILSON \& ROOM, 1983; RIBEIRO et al., 1990, PERRUSO \& CASSINO, 1997).

O presente trabalho teve como objetivo avaliar o método de amostragem presença-ausência para $\boldsymbol{D}$. notus, visando-se à obtenção de alguns valores referenciais que tornem esta metodologia de fácil aplicação no estabelecimento do Manejo Integrado de Pragas na cultura de erva-mate.

\section{MATERIAL E MÉTODOS}

Os dados foram coletados em área comercial não tratada com produtos fitossanitários, com sistema de cultivo a pleno sol, formada por plantas de ervamate de aproximadamente dez anos de idade cultivadas no espaçamento 4,0 x 1,5m, implantada no município de Cascavel, na região oeste do Estado do Paraná.

O trabalho foi realizado de abril de 1999 a julho de 2000. As amostras foram coletadas quinzenalmente, avaliando-se dez plantas escolhidas aleatoriamente, retirando-se 24 folhas de cada planta. As amostras foram coletadas em diferentes pontos das plantas, sendo que, para isto, as plantas amostradas foram divididas verticalmente em três estratos (inferior, médio e superior), e cada estrato, ou terço da planta, foi dividido em quatro partes, coincidindo com quadrantes com sentidos norte, sul, leste e oeste. Em cada quadrante de cada estratos foram coletadas duas folhas, uma jovem outra madura, sendo consideradas folhas jovens aquelas mais claras, tenras e emitidas na primavera anterior, e folhas maduras aquelas mais escuras, endurecidas e emitidas duas primaveras antes. As folhas foram etiquetadas, acondicionadas em sacos plásticos, levadas ao laboratório e observadas sob microscópio estereoscópico com aumento de 20 vezes. Durante a avaliação, também foram realizadas contagens de outros ácaros presentes nas folhas. A espécie estudada foi identificada por comparação com lâminas depositadas no laboratório de Zoologia da UNIOESTE com exemplares identificados pelo Prof. Dr. Carlos H. W. Flechtmann (ESALQ/USP).

Para se avaliar a distribuição espacial do ácaro no erval, visando-se à amostragem binomial (presença-ausência), determinaram-se a variância ( $\left.\mathrm{S}^{2}\right)$ e o número médio de ácaros por folha (X) em cada data de amostragem. Com base nestes dados, utilizou-se a Lei da Potência de Taylor (TAYLOR, 1961; WILSON et al., 1983; RIBEIRO et al., 1990; PERRUSO \& CASSINO 1997), que descreve a relação entre a variância e a média das densidades populacionais obtidas como S2 = a.Xb. Os coeficientes a (fator de amostragem) e b (índice de agregação da espécie) da Lei da Potência $\left(S^{2}=a \cdot X^{b}\right)$ foram estimados por análise da regressão como: $\ln \left(\mathrm{S}^{2}\right)$ $=\ln (a)+b \cdot \ln (X)$. Em seguida, foi avaliada a relação entre a proporção de folhas infestadas com pelo menos um ácaro e o número médio de ácaros por folha utilizando-se o modelo matemático proposto por WILSON \& ROOM (1983), representado pela equação a seguir, na qual $\hat{p}$ é a proporção de folhas infestadas com o ácaro, X é o número médio de ácaros por folha, e a e b são os coeficientes de Taylor. A qualidade do ajustamento do modelo foi avaliada pela análise da regressão da proporção estimada ( ) de folhas infestadas e a proporção de folhas infestadas ( EMBED Equation.3 ) observadas no campo.

$$
\hat{p}=1-\exp \left(-\frac{X}{a X^{b-1}} \ln \left(a X^{b-1}\right)\right)
$$

$\mathrm{O}$ número total de folhas requeridas $\mathrm{n}$ foi determinado em função da densidade média, a partir da equação (WILSON et al. 1983): $n=\left(\frac{z_{\beta}}{D}\right)^{2} \frac{p}{\hat{p}} \quad$ em que

D corresponde ao erro admitido (neste trabalho, foram escolhidos $\mathrm{D}=0,1$ e $\mathrm{D}=0,2$ ), e $p$ é a probabilidade de não serem encontrados ácaros nas folhas com $p=1-\hat{p}$. Por fim, $z_{\beta}$ éo valor da variável $z$, para o qual $Z(z)=2 \beta$, sendo:

$$
Z(z)=\int_{z}^{\infty} \varphi(t) d t
$$

em que $\varphi(t)$ é a função de freqüência normal:

$$
\varphi(t)=\frac{1}{\sqrt{2 \pi}} e^{-t^{2} / 2}
$$

$\mathrm{Z}(\mathrm{z})$ pode ser reescrito em termos da função erro erf(z), 
como:

$$
Z(z)=\frac{1}{2}\left(1-\operatorname{erf}\left(\frac{Z}{\sqrt{2}}\right)\right)
$$

Neste trabalho, foi escolhido $\beta=0,05$, o que implica $\mathrm{z}_{\mathrm{\beta}} \cong 1,28155156$, sendo que $\mathrm{z}_{\mathrm{B}}$ foi calculado via métodos numéricos.

\section{RESULTADOS E DISCUSSÃO}

Durante o período de avaliação, $\boldsymbol{D}$. notus apresentou uma densidade populacional média de 5,311 ácaros por folha, sendo que as médias observadas abrangeram de 0,021 a 35,167 ácaros por folha.

A distribuição espacial de $\boldsymbol{D}$. notus foi agregada, tendo-se como base o valor estimado de $\mathrm{b}=$ 1,372 (Figura 1), significativamente maior que 1 pelo teste $t$, conforme proposto por SILVEIRA NETO (1976), sendo o valor de $\mathrm{a}=34,491$ considerado elevado (ZALOM et al. 1985; WILSON 1985). A presença de predadores diminui a agregação de ácaros fitófagos (RIBEIRO 1990) e, no presente trabalho, durante a contagem dos ácaros, observou-se rara ocorrência de predadores. Assim, o padrão de distribuição em regiões com diferentes condições e com a presença freqüente de predadores deve ser reavaliado.

Outro dado que confirma o comportamento agregado de $\boldsymbol{D}$. notus são os valores dos pontos da curva, estimados pelo modelo proposto por WILSON \& ROOM (1983), com base no número médio de ácaros por folha, e a relação variância/média, que aumentaram com o aumento da densidade média de ácaro por folha (Figura 2) (WILSON, 1985).

O ajuste do modelo utilizado, avaliado pela análise da regressão entre a proporção de folhas infestadas estimadas e observadas (Figura 3), resultou em $\mathrm{r}^{2}=0,72$, o que se considera uma boa indicação de que a proporção de folhas infestadas estimadas pode ser usada como indicadora do número médio de ácaros por folha.

O número de amostras (folhas) requeridas em função da densidade média com um nível de precisão de $10 \%(\mathrm{D}=0,1)$ foi muito elevado em baixas densidades (Figura 4). No entanto, adotando-se $\mathrm{D}=0,2$, ocorre uma redução considerável no número de amostras requeridas, viabilizando sua aplicação prática. A partir de cinco ácaros por folha, é necessário serem amostradas 103 folhas, um número pequeno para se avaliar apenas a presença ou não do ácaro (Tabela 1). São necessários mais estudos visando-se a identificar o nível populacional em que devem ser iniciadas as ações para se evitar que sejam provocados danos econômicos à cultura.

A amostragem de $\boldsymbol{D}$. notus na cultura da erva-mate por meio da contagem de indivíduos foi

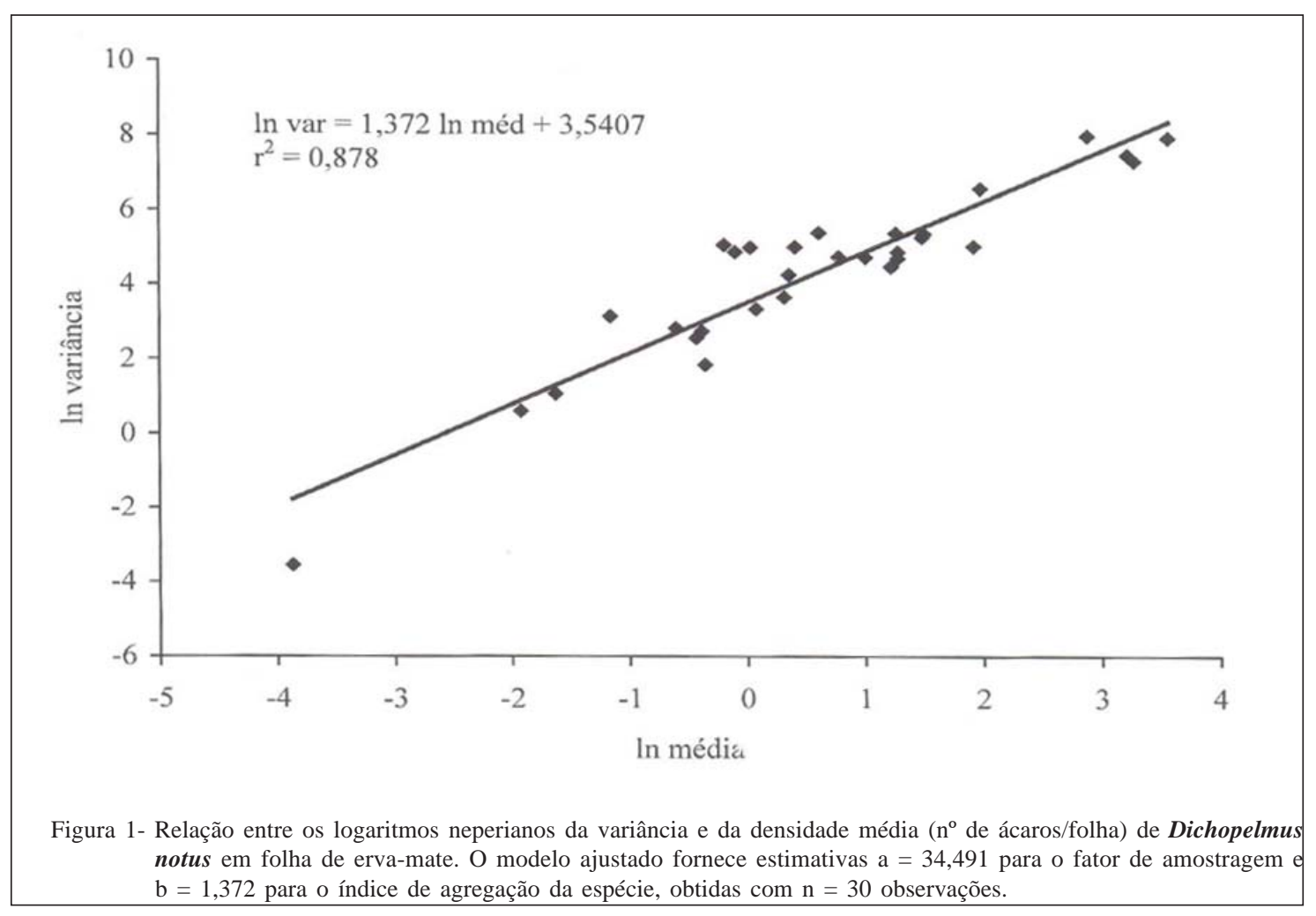

Ciência Rural, v.38, n.3, mai-jun, 2008. 


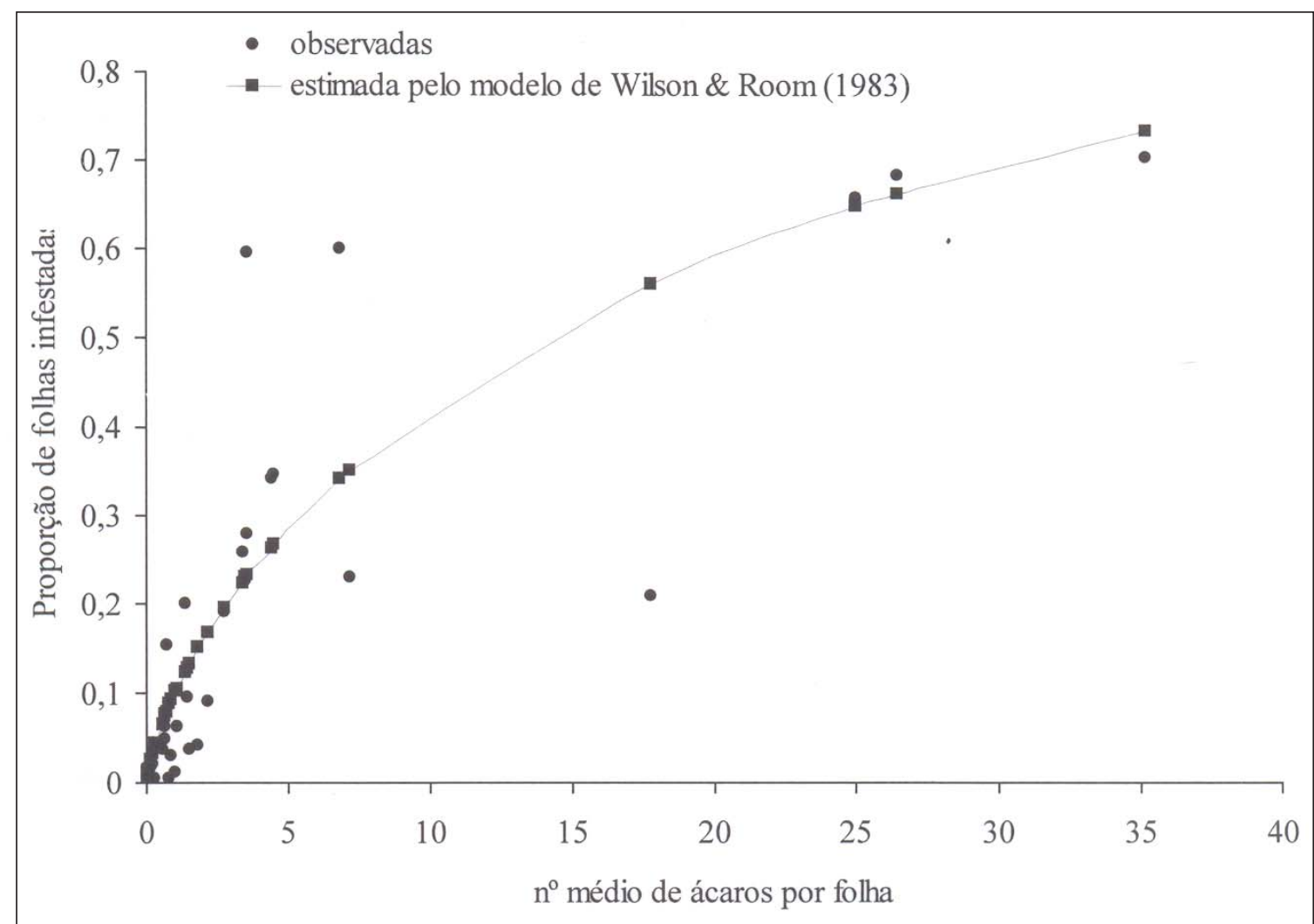

Figura 2- Relação entre a proporção de folhas infestadas e o número médio de Dichopelmus notus por folha em erva-mate.

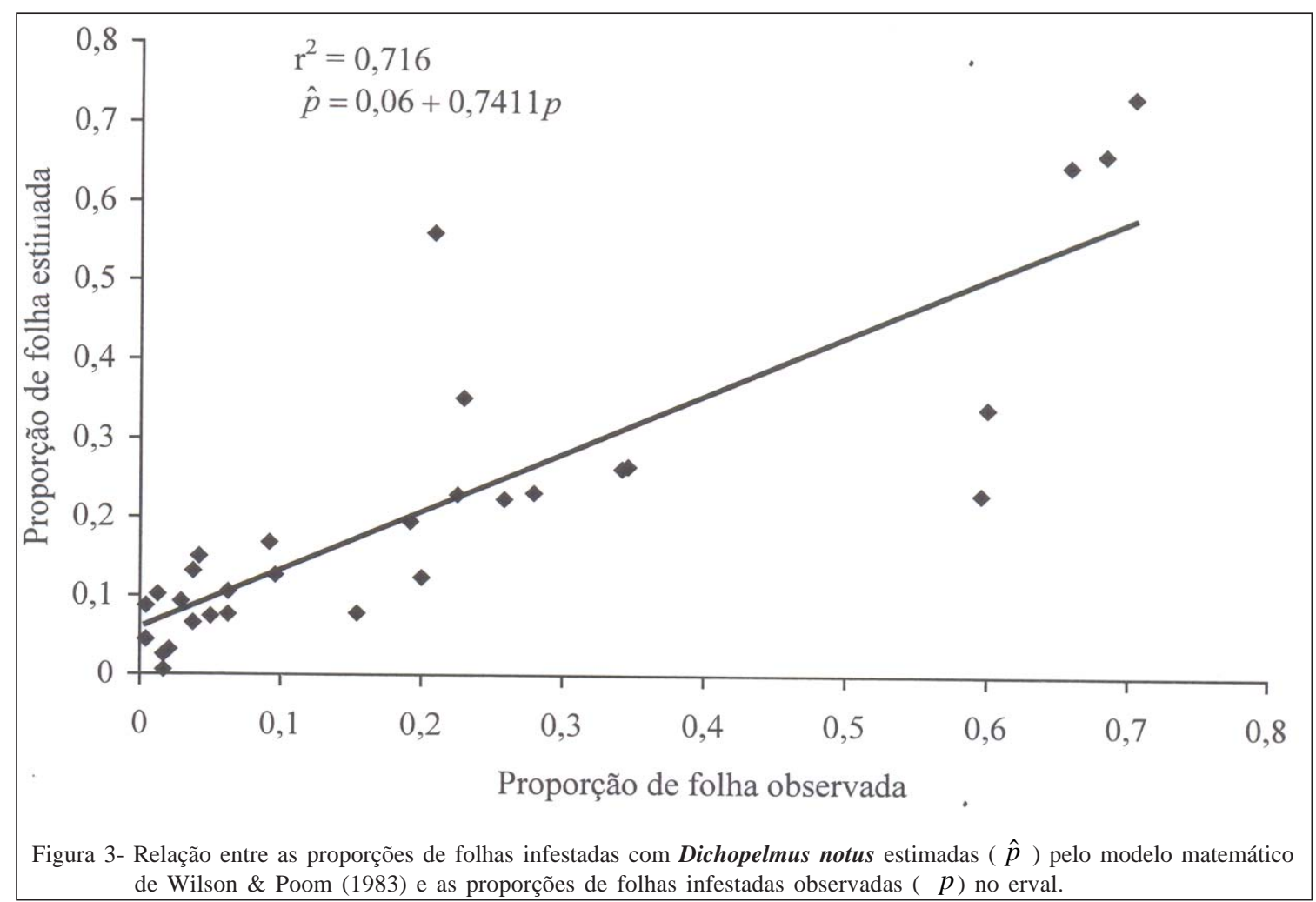

Ciência Rural, v.38, n.3, mai-jun, 2008. 


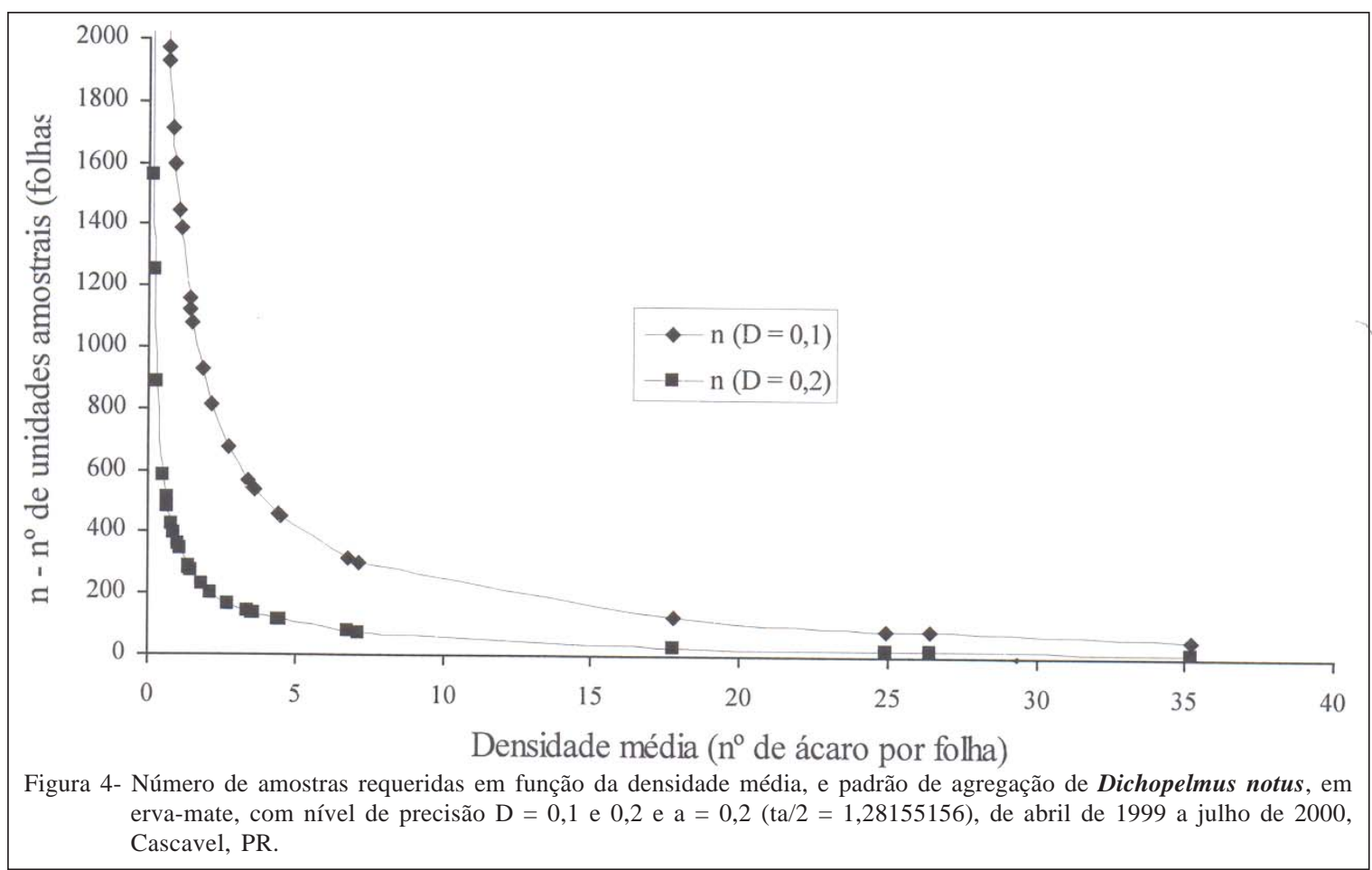

Tabela 1 - Número total de folhas requeridas em função da densidade média e do padrão de agregação de Dichopelmus notus, em erva-mate, com nível de precisão $\mathrm{D}=0,1$ e 0,2 .

\begin{tabular}{lcc}
\hline & & \\
& & \\
Densidade média $^{1}$ & D $=0,1$ & D $=0,2$ \\
\hline 90 & 14 & 3 \\
80 & 17 & 4 \\
70 & 22 & 5 \\
60 & 28 & 7 \\
50 & 37 & 9 \\
40 & 50 & 12 \\
30 & 72 & 18 \\
20 & 114 & 28 \\
10 & 225 & 56 \\
9 & 248 & 62 \\
8 & 275 & 69 \\
7 & 310 & 77 \\
6 & 354 & 88 \\
5 & 413 & 103 \\
4 & 496 & 124 \\
3 & 627 & 157 \\
2 & 864 & 216 \\
1 & 1473 & 368 \\
0,5 & 2485 & 621 \\
\hline & &
\end{tabular}

${ }^{1}$ Número de ácaros por folha.

${ }^{2}$ Número de plantas amostradas $\mathrm{x}$ número de folhas por planta. proposta por VIEIRA NETO \& CHIARADIA (1999), sendo necessário, por este método, segundo os autores, serem examinadas 330 folhas. A aplicação desta metodologia, no entanto, pode ser desencorajada pelo alto número de amostra requerido e pela necessidade de utilização de microscópio estereoscópico.

Pelo constatado no presente trabalho, o baixo número de amostras requeridas, utilizando-se $\mathrm{D}=0,2$, torna o monitoramento populacional de $\boldsymbol{D}$. notus através do método presença-ausência, com simples observação de a folha estar infestada ou não, com grande potencial de aplicação prática. Este método de amostragem apresenta as vantagens de ser simples, fácil, rápido e também mais barato, além de permitir que o erval seja dividido em talhões menores, o que permitirá detectar focos iniciais.

\section{CONCLUSÃO}

A proporção de folhas infestadas estimadas pelo modelo de WILSON \& ROOM (1983) mostrou ser uma medida confiável para se estimar a densidade populacional de Dichopelmus notus. Além disso, o número baixo de folhas a serem amostradas utilizandose $\mathrm{D}=0,2$ confirma a viabilidade do uso do método de 
amostragem presença-ausência desenvolvido para se estimar a densidade populacional de $\boldsymbol{D}$. notus em ervamate.

\section{AGRADECIMENTOS}

Os autores agradecem ao Conselho Nacional de Desenvolvimento Científico e Tecnológico (CNPq), pela concessão da bolsa de Produtividade em Pesquisa.

\section{REFERÊNCIAS}

COLL, O.R.; CACERES, M.S. Determinación de la flutuación poblacional del "ácaro del bronceado" de la yerba mate y sus enemigos naturales. In: WINGE, H. et al. Erva-mate: biologia e cultura no Cone Sul. Porto Alegre: UFRGS, 1995. p.121-128.

GOUVÊA, A. et al. Dinâmica populacional de ácaros (Acari) em plantas de erva-mate (Ilex paraguariensis St. Hil.: Aquifoliaceae). Neotropical Entomology, Londrina, v.35, n.1, p.101-111, 2006.

MENEGUZZI, Z. et al. Tamanho ideal do talhão para amostragem do ácaro-do-bronzeado em erva-mate. Acta Ambiental Catarinense, Chapecó, v.1, n.2, p.49-55, 2002.

PARSEVAL, M. Von. Eriofídas no Brasil. Revista Agronômica, Porto Alegre, v.3, n.30, p.511-517, 1939.

PERRUSO, J.C.; CASSINO. P.C.R. Plano de amostragem presença-ausência para Selenaspidus articulatus (Morg.) (Homóptera: Diaspididae) na cultura de laranja. Anais da Sociedade Entomológica do Brasil, Jaboticabal, v.26, n.2, p.321-326, 1997.

RIBEIRO, L.G. et al. Plano de amostragem presença-ausência para Panonychus ulmi (Kock, 1836) (Acari: Tetranychidae) em macieira. Anais da Sociedade Entomológica do Brasil, Jaboticabal, v.19, n.1, p.211-220, 1990.
SANTANA, D.L.Q. et al. Principais características de três espécies de ácaros em erva-mate, no sul do Brasil. Colombo: Embrapa Floresta, 1997. 2p. (Comunicado Técnico, 17).

SILVEIRA NETO, S. et al. Manual de ecologia dos insetos. Piracicaba: Ceres, 1976. 419p.

TAYLOR, L.R. Aggregation, variance and the mean. Nature, London, n.189, p.732-735, 1961.

VIEIRA NETO, J.; CHIARADIA. L.A. Amostragem de Dichopelmus notus Keifer (Acari, Eriophyidae) na cultura da erva-mate. Pesquisa Agropecuária Gaúcha, Porto Alegre, v.5, n.2, p.357-361, 1999.

WILSON, L.T. Estimating the abundance and impact of arthropod natural enemies on IPM systems. p.303-322. In: HOY, M.A.; D.C. HERZOG. Biological control in agricultural IPM systems. New York: Academic, 1985. p.303-322.

WILSON, L.T. et al. Within - plant distribution of spider mites (Acari: Tetranychidae) on cotton: a developing implementable monitoring program. Environmental Entomology, Lanhan, v.12, n.1, p.129-134, 1983.

WILSON, L.T.; P.M. ROOM. Clumpling patterns of fruit and arthropods in cotton, with implications for binomial sampling. Environmental Entomology, Lanhan, v.12, n.1, p.50-54, 1983.

ZALOM, F.G. et al. Distribution of Panonychus ulmi (Mc Gregor) and Euseius tularensis Congdon on central Califórnia orange trees with implications for binomial sampling. Agriculture, Ecosystems \& Environment, Amsterdam, n.14, p.119-129, 1985. 